\title{
The Research of Velocity Compensation Method Based on Range-Profile Function
}

\author{
Bao Han \\ School of Software \\ University of Science and Technology Liaoning, Anshan, 114000, China
}

sky46bh@126.com

\begin{abstract}
In the high-resolution radar imaging system, step-frequency radar signal is very important in getting the high-resolution range-profile, which can be used for target's detection and recognition. However, target radial velocity will produce many problems, therefore, movingtargets imaging is a bottleneck for step-frequency radar signal. In this paper, an effective motion compensation methods is presented, which is based on the functon of Range-profile Contrast. The numeric simulation indicates that this method is effective, which can estimate the target's velocity in real-time, and it is easy to be used in engineering project. After target's motion compensation, the high-resolution range-profilewill be much better than that is used to be, which can be used for the detection, recogution and ranging of moving targets..

Keywords: Step-Frequency; Range-profile; Velocity Estimation; Motion Compensation; Range-profile Contrast

\section{Introduction}

In radar system, the pulse compression signals are widely used in getting the highresolution range-proffle and it is called as HRRP in short. Step-frequency (SF) radar signal is one of the most importan pulse compression signal, which is well illustrate in reference [1 3]. For the processing of step-frequency radar signal, the velocity between target and radar will make the distortion of HRRP. Therefore, for the non- cooperative targets, the step-frequency radar signal has a serious problem of Range-Doppler coupling [5].

The impact of Range-Doppler coupling can be eliminated or relieved target's by motion compensation. Suppose, the target's velocity is precisely estimated, the phase terms which is caused by the motion of target, can be compensated by removing it. Many motion compensation methods are presented based on velocity estimation. In reference [6, 7], based on time domain and waveform entropy, two motion compensation methods are presented, however they have low estimation accuracy in low SNR, and they are only effective for moving targets with low velocity. In reference [8], based on SF and Pulse Doppler system, an effective method of motion compensation is presented; numeric simulation shows that this new method has higher compensation accuracy and better anti-noise performance. But it will increase the calculation complexity of radar system. Until now, except for those methods mentioned above, other effective motion compensation methods have been also presented, for example in the reference [9-11], which have gotten much better compensation result.
\end{abstract}


But, in common, they need much larger computation complexity and calculation amount, meantime they are not easy to be used in project application.

As discussed above, in low SNR, the motion compensation method of moving targets is still a key problem for SF signal. Furthermore, the application in project also should be considered. In this paper, based on the analysis of range-profile, an effective motion compensation method is presented. Numeric simulation indicates that this method has a high estimation accuracy and low calculated amount than that used to be, which is easy to be used in engineering project application.

\section{The Range-Doppler Coupling of SF Radar Signal}

Step-frequency radar signal is a pulse sequence that the carrier frequency increased by the fixed step length, which is shown in formula (1).

$$
u(t)=\frac{1}{\sqrt{N T_{1}}} \sum_{i=0}^{N-1} \operatorname{rect}\left(\frac{t-i T_{r}}{T_{1}}\right) \exp j 2 \pi\left(f_{0}+i \Delta f\right) t
$$

Where, $f_{0}$ is the centre frequency, $T_{1}$ is the pulse widh, $T_{r}$ is the pulse repetition cycle, $\Delta f$ is the step-frequency value, $N$ is the pulse number.

Suppose, the distance between radar and target is $R_{0}$, after $I / Q$ frequency mixing of double channel, we can get the complexenvelop outpor of echo signal:

$$
G_{i}=\exp \left[-j 2 \pi f_{i}\left(\frac{2 R}{C}\right)\right] \Rightarrow \exp \left(j \psi_{i}\right)
$$

Suppose, the target is moving, the phase term of target echo signal can be expressed as follows:

$$
\mathbb{W}_{i}=-2 \pi f_{i} \frac{2(R-v t)}{\mathrm{c}}
$$

Suppose, is relatively radar target of radial velocity, $t$ is time, which is shown as follows:

$$
t=i T_{r}+\frac{T_{1}}{2}+\frac{2 R}{\mathrm{c}}
$$

Introducing the formula (4) into (3), the formula (5) can be gotten

$$
0 \psi_{i}=\frac{2 f_{0} R}{\mathrm{c}}+\underbrace{\frac{2 n R \Delta f}{\mathrm{c}}}_{\text {const }}-\underbrace{\frac{2 f_{0} v T_{r} n}{\mathrm{c}}}_{f_{R}}-\underbrace{\frac{2 v \Delta f T_{r} n^{2}}{\mathrm{c}}}_{\text {Spread }}
$$

The Eq.(5) reveals the special properties of SF radar signal. The first term represents a constant phase migration. The second term is produced by the product of frequency. The third term $f_{d}$ in Eq. (5) is a linear phase term, which represents the Doppler frequency migration due to target motion. The processing of IDFT mistakes the Doppler frequency as a frequency migration due to range and thus results in the shifting of target range from its true range. 
According to the literature [5], suppose, in order to perfectly compensate LPT, the sustainable error of distance measurement is half of range resolution of SF signal, which is shown as follows:

$$
\Delta R=\frac{\Delta r}{2}=\frac{\mathrm{c}}{4 N \Delta f}
$$

Then, the estimation accuracy of target speed must satisfy Eq. (7).

$$
|\Delta v|_{\mathrm{LPT}} \leq \frac{\mathrm{c}}{2}\left(\frac{\Delta f}{f_{0}}\right)\left(\frac{1}{T_{r}}\right)\left(\frac{1}{N \Delta f}\right)=\frac{\mathrm{c}}{4 f_{0} N T_{r}}
$$

The fourth term 'Spread' in Eq. (5) is a quadratic phase term, which causes by the frequency spread. According to the literature [5], suppose, the sustainable change of quadratic phase term satisfies the following requirement.

$$
2 \pi \frac{2 N \Delta f|\Delta v|_{\mathrm{QPT}}}{\mathrm{c}} N T_{r} \leq \frac{\pi}{2}
$$

Then, the estimation accuracy of target speed must satisfy Eq. (9)

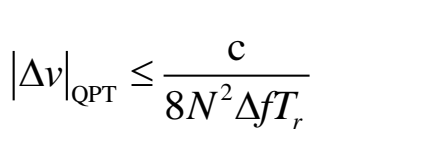

From the above analysis we can know hat, target motion is the main reason of rangeprofile variation for step-frequency. Therefore important feature of the target motion can be extracted by analyzing the characteristics of the range-profile.

\section{Velocity Estimation Method bassed on Range-profile Contrast}

The contrast function of range-profile is the ratio of the goal and the surrounding environment brightness difference it is a measure of waveform focus degree. In the range-profile the defocus brought by movement will make the amplitude of scattered points around the mean and the contrast ratio is very low. However, the degree of the waveform focusing can be mproved by motion compensation.

By the concept of contrast function, it is easy to understand that contrast function is the degree of disorder, the greater contrast function value is, and the lower disorder range-profile is. And when the range-profile is focused, the contrast function will have the maximum value. The range-profile contrast function is defining as follow:

The contrast function of range-profile can be defined as following:

$$
\begin{gathered}
C_{1}(v)=\max \{H\} \\
C_{2}(v)=\frac{\sqrt{A\left\{[H-A\{H\}]^{2}\right\}}}{A\{H\}}
\end{gathered}
$$


Or

$$
C_{3}(v)=\frac{\sqrt{A\left\{\left[H^{2}-A\left\{H^{2}\right\}\right]^{2}\right\}}}{A\left\{H^{2}\right\}}
$$

The steps of Maximum range-profile contrast method are shown as follows.

(1) calculating the motion compensation factor by the initial estimated value of velocity:

$$
p_{i}=\exp \left[-j 2 \pi\left(f_{0}+i \Delta f\right) \frac{2 v_{0} i T_{r}}{\mathrm{c}}\right]
$$

(2) compensating the echo signal:

$$
G_{i}^{\prime}=G_{i} p_{i}=\exp \left[-j 2 \pi\left(f_{0}+i \Delta j\right) \frac{2\left(R_{0}-\Delta v i T_{i}\right)}{c}\right]
$$

(3) making the inverse Fourier transform for compensated signal:

$$
h(l)=\sum_{i=1}^{N} G_{i} \exp \left[2 \pi \frac{a i}{N}\right]
$$

(4) calculating the range-profile contrast fluction according to formula (11).

(5) confirming the next estimated vetocity value by a certain optimization method and repeat the steps till the maximum value of range-profile entropy is found, and then the final estimated velocity can be gotten

\section{The Simulation of Estimation Result}

Supposing the targe model is a rigid body (the radial velocity of every scatters are the same), which is shown in Figure 1. The target is composed by four strong scattering points which are parted to $7 \Delta r, 3 \Delta r$ and $6 \Delta r$ in sequence and noted as $A, B, C$, and $D$ respectively. The normalized scattering intensity ratio is $1: 10: 6: 8$, the radial distance $1 \mathrm{~km}$. The parameters of the step-frequency radar signal transmitted by radar is as follows: transmitting frequency $f_{0}=94 \mathrm{GHz}$, step value $\Delta f=4 \mathrm{MHz}$, pulse width $T=100 \mathrm{~ns}$, repeat cycle $\operatorname{Tr}=20 \mathrm{u}$, pulse number $N=128, \Delta r=c /(2 N \Delta f)=0.3 \mathrm{~m}$ (The parameters are adopted in all the simulation below). 


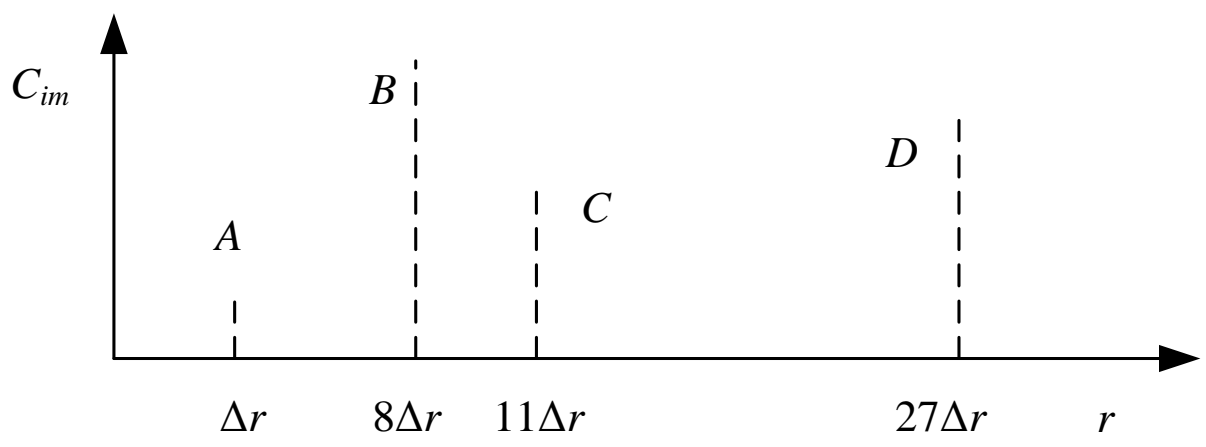

Figure 1. Point of the strong scattering target

Figure 2 is the velocity estimation characteristic figure which is dray $n$ by the formula (11). From the figure we can know that the contrast function of range-profile have some local maximum points, which requires optimizing search algorithm to ensure jump out of the local maximum point and converges to the true value.

The Figure 3 shows the velocity estimation error with different SNR through 1000 times' Monte Carlo simulation experiments. From the picture we canknow that no matter how high the SNR of echo is, the maximum range-profile contrast ratio can't achieve high accuracy of compensation. Therefore, it can only be used to compensate for coarse profile. And, we can't achieve better compensation effect because the standard deviation of the velocity estimation errors and the discrete degree of the celocity estimation error is big. According to Figure 4, the velocity estimation error alsoproves the aboveconclusion.

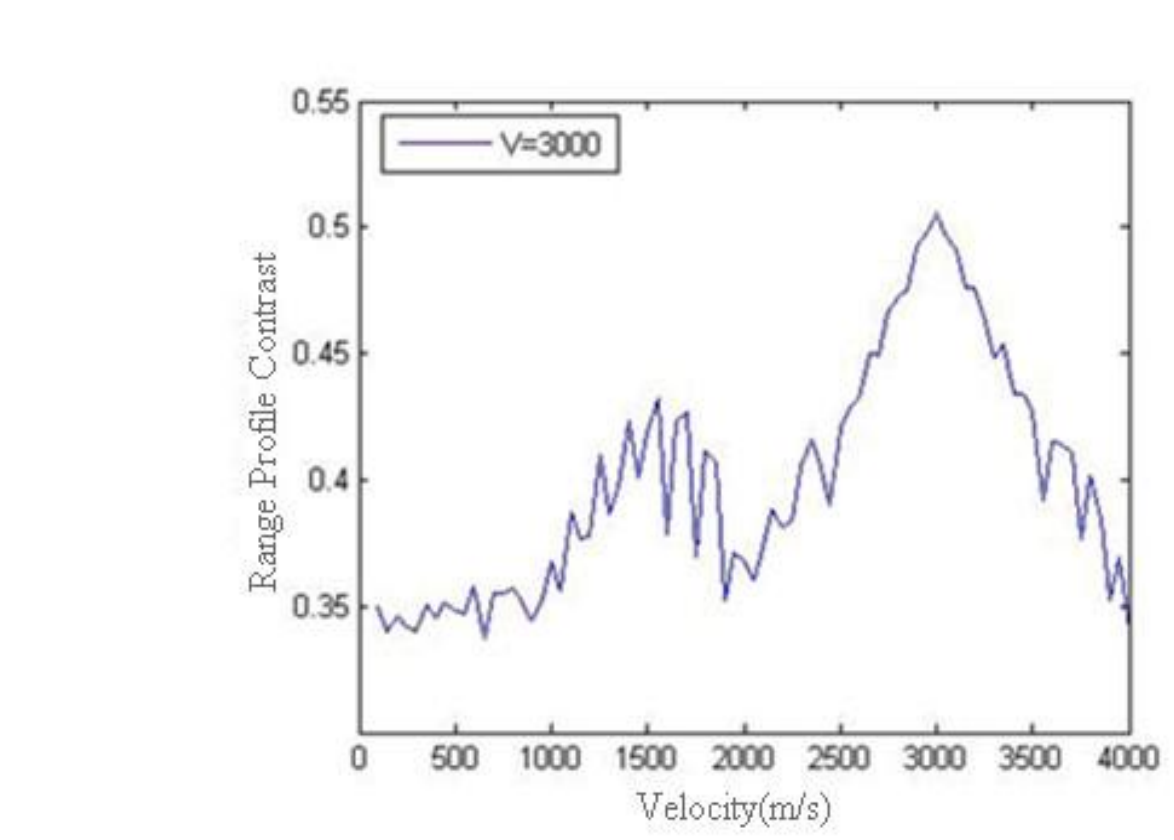

Figure 2. The characteristic figure of elocity estimation 

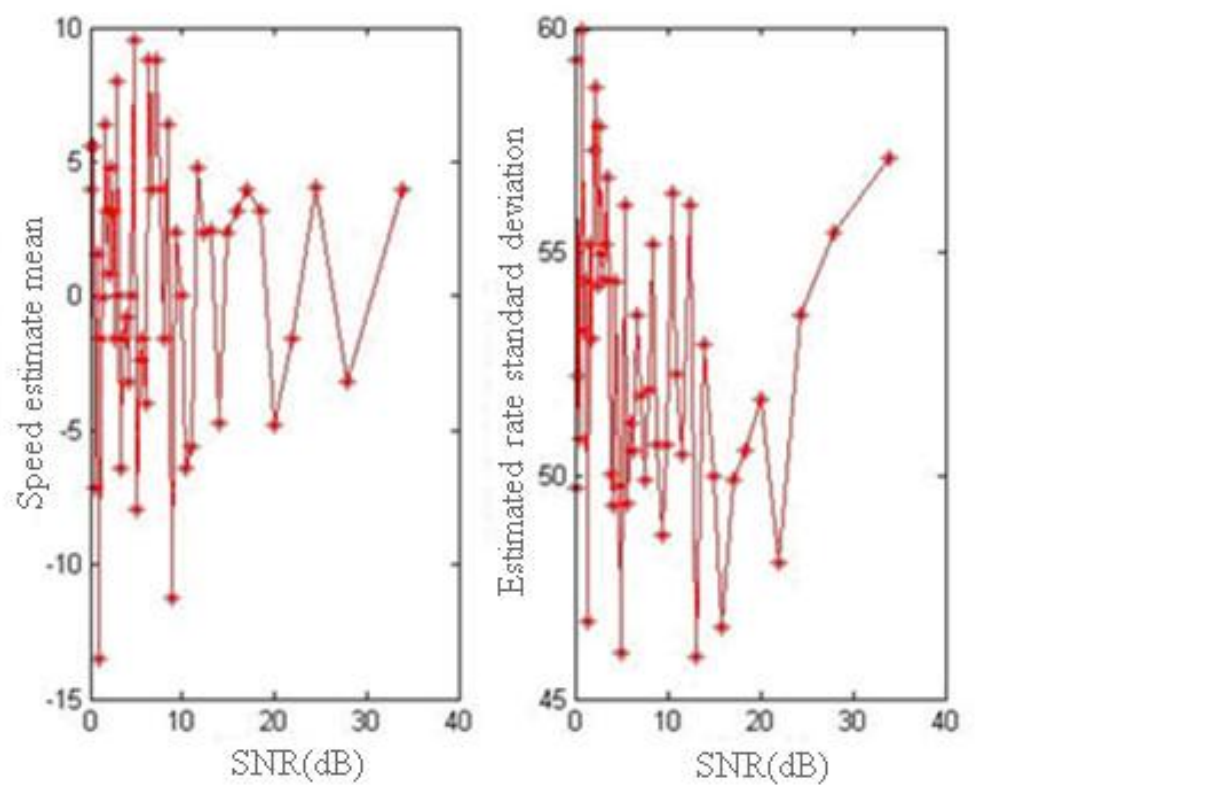

a) The mean error of estimation

b) Standard deviation error of estimation

Figure 3. Velocity estimation error with the signal-to-noise ratio variation

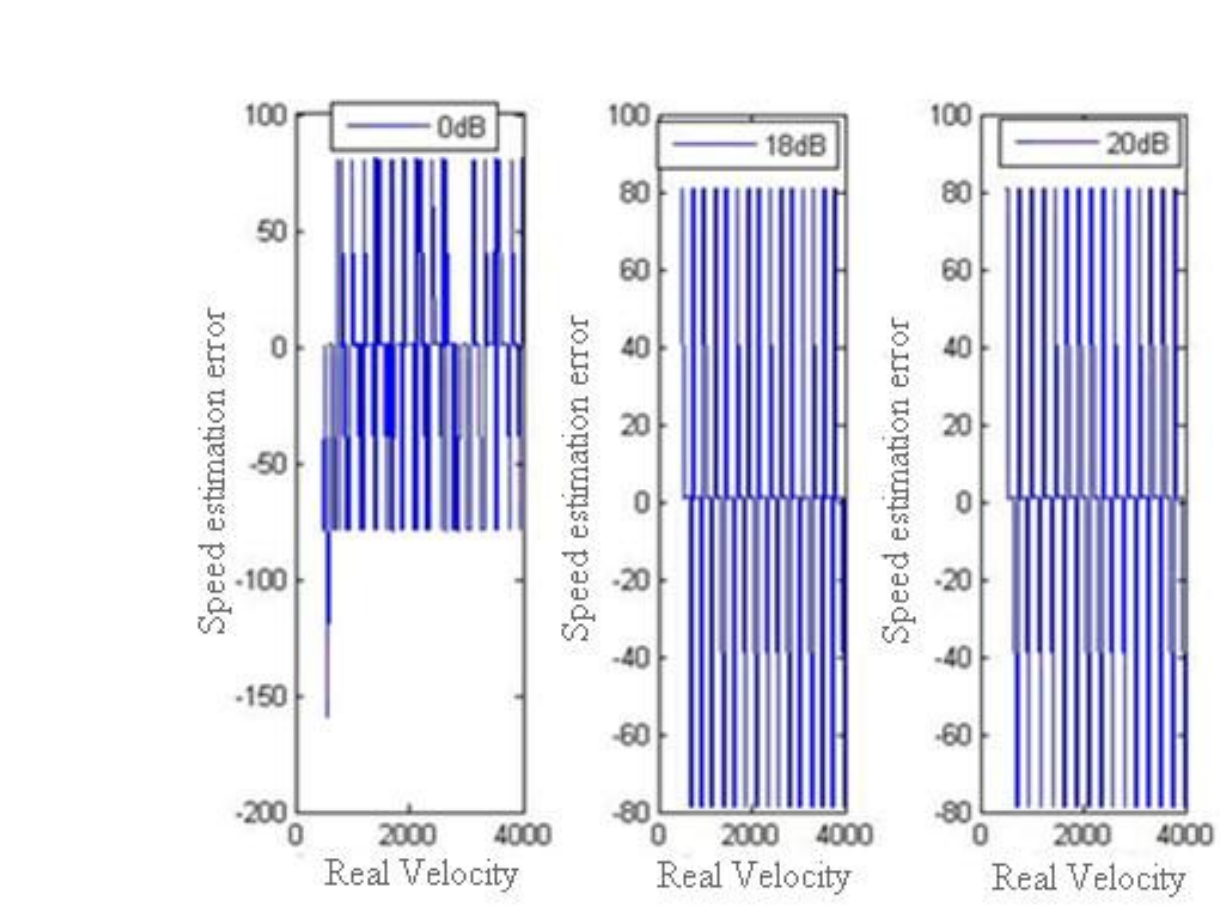

Figure 4. The error chart of elocity estimation 


\section{Conclusions}

According to the discussion above, we can get several conclusions as follows:

(1) This velocity estimation method has the global optimal value and local optimal value, therefore the initial value and optimization search method will make a great influence on the estimate effect.

(2) The estimation effect of the maximum range-profile contrast method can only be used for a coarse estimate in low SNR, but when the SNR is increasing, the accuracy of estimation become better.

Based on the discussion above, maximum range-profile contrast method is suitable for the engineering application, and the following research is looking for simple initial velocity estimation algorithm. It should be ensure to choose the estimated range which can cover the true value, and do not contain local optimal value. Then, the suitable evaluation function and optimization search algorithm should be given. At the same time, it mus be reduce the computational complexity and improve the velocity estimation precision.

\section{References}

[1] D. R. Wehner, "High-resolution Radar. Norwood", MA: Artech House, (1995).

[2] T. H. Einstein, "Generation of High-resolution Radar Range-profiles and Range-profile Autocorrelation Functions using Stepped Frequency Pulse Trains”, MT, Lincoln Lâb, Lexington, MA, Project Rep, TT-54, (1984) October.

[3] H. Wu, X. Li and Y. Chen, et al., "Spatial-Temporal AdaptiveClutter Classification Suppression and Dim Small Moving Targets Detection", Journal of Infrared and Millimeter Waves, vol. 25, no. 4, (2006), pp. 301305.

[4] C. -G. Sun and X. -G. Li, "Two-step Streteh Processing Method for MMW High-Resolution Radar", Journal of Infrared and Millimeter Waves, vol 22, no. 6, (2006) pp. 457-460.

[5] T. Long, "Doppler Performance Analysis of Frequency Stepped Radar Signal", Modern Radar, vol. 2, (1996), pp. 31-37.

[6] J. Liu, X. -G. Li and W. Wu Application of Waveform Entropy Method for Motion Compensation of MMW Costas Frequency Hopped Radar”, Journal of Infrared and Millimeter Waves, vol. 22, no. 4, (2003), pp. 303306.

[7] G. Wang and X-O. Li, "Compound Approach of Measuring Velocity Based On Step-Frequency And Pulsedoppler System", Journal of Infrared and Millimeter Waves, vol. 27, no. 3, (2008), pp. 191-194.

[8] F. Berizzi, M. Martorella, A. Cacelamano and A. Capria, "A Contrast- Based Algorithm For Synthetic RangeProfile Motion Compensation, IEEE TRANSACTION ON GEOSCIENCE AND REMOTE SENSING, vol. 46, no. 10, (2008), pp 3053-3062.

[9] G. -F. Xia, H. -Y. Su and P. -K. Huang, "Motion Compensation Methods for LPRF Modulated Frequency step-frequency (MFSF) radar", Journal of Systems Engineering and Electronics, vol. 21, no. 5, (2010), pp. 746-751.

[10] H. -B. Li and J. -D. Lu, "Target Motion Compensation Algorithm Based on Keystone Transform for Wideband Pulse Doppler Radar”, Transactions of Beijing Institute of Technology, vol. 32, no. 6, (2012), pp. 626-63

[11] Y. Lin and X. Si, "Motion Compensation of Stepped Frequency Signal Based on Evaluation Function", AERO W EAPONR, vol. 3, (2009), pp. 31-35. 
International Journal of Hybrid Information Technology

Vol.7, No.2 (2014)

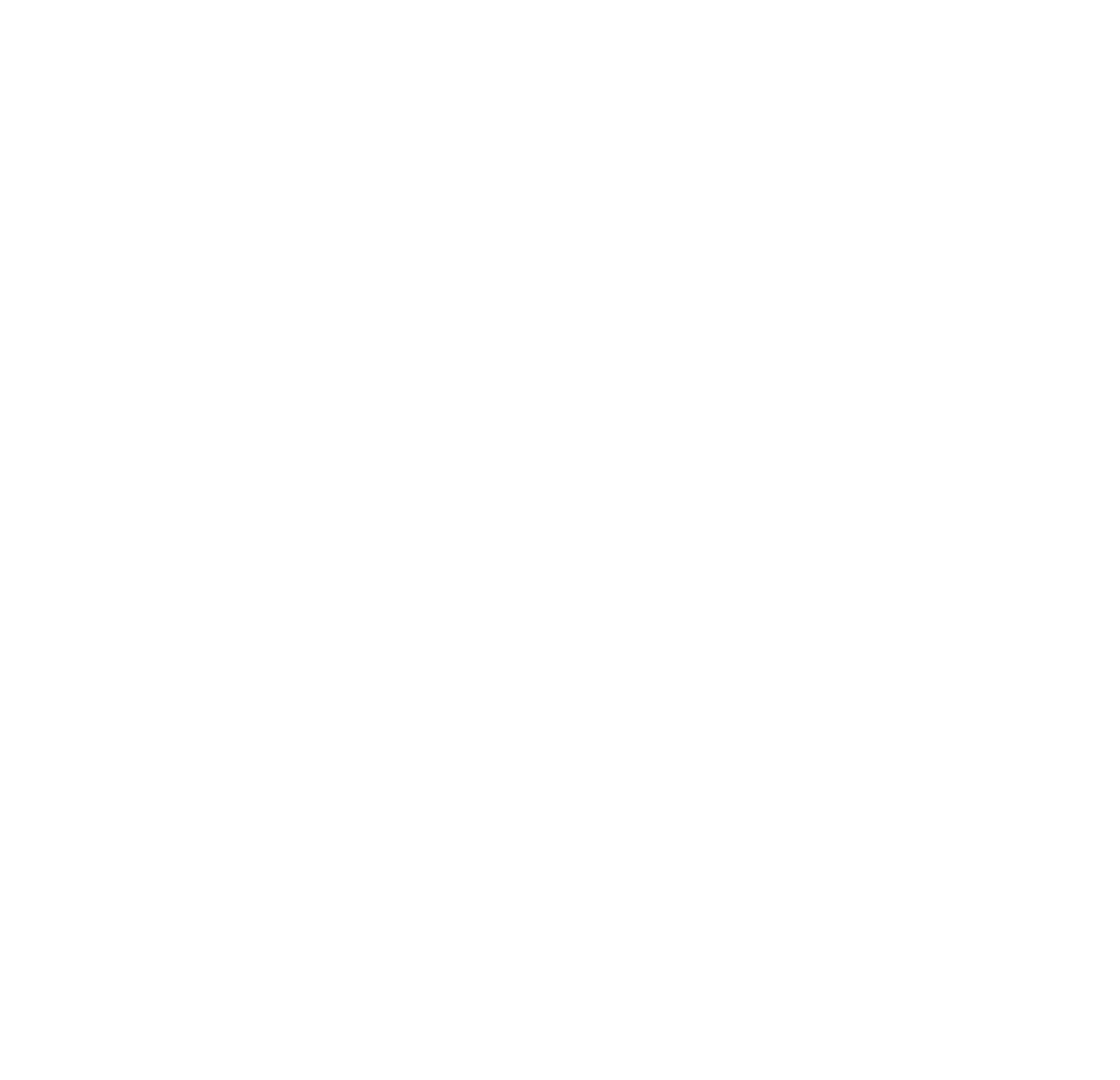

